\title{
Yield of intermediate products in the drought process of wheat milling
}

\author{
Yevgen Kharchenko1, Andrii Sharan', \\ Olena Yeremeeva ${ }^{2}$, Larysa Novak ${ }^{2}$
}

1 - National University of Food Technologies, Kyiv, Ukraine,
2 - Uman's National University of Gardening, Uman, Ukraine

\begin{tabular}{l} 
Keywords: \\
Wheat \\
Flour \\
Milling \\
Roller \\
Yeald \\
\hline
\end{tabular}

Article history:

Received 05.08.2017

Receivedin revised

form 11.11.2017

Accepted 29.12.2017

Corresponding

author:

Yevgen Kharchenko

E-mail:

a-537@ukr.net

DOI:

$10.24263 / 2304-$

974X-2017-6-4-3

\section{Abstract}

Introduction. In order to determine the yield of intermediate milled products, the process of crop formation during the milling of wheat into varietal flour has been investigated.

Materials and methods. On the first three break systems, intermediate milling products under the rollers were selected and sifted to determine the mode of operation of the systems, and then pass fractions were sieved on sieves to determine the yield of individual product fractions. The results of the research were presented as a dependence of "general product - yield fraction".

Results and discussion. The output of all milling products on the first break system, depending on the milling mode, is nonlinear. On the second break system, the only dependence on the yield of small middlings and superfine flour is linear, and the yield of large and medium middlings, as well as flour has a nonlinear character. On the third break system, the large dependence of the yield of products on their milling regimes, all milling products except the yield of the small middlingshave a nonlinear character. On the third break system, the small linear dependencies of the yield of products from the milling regime are only superfine flour and flour, the remaining products are nonlinear in nature.

With an increase in the total product of intermediate shredding products from $29,4 \%$ to $56,6 \%$, on the 1 st system there was an extremum of the output of large grains at $40,0 \%$. On the second tread system, with an increase in the total product of intermediate shredding products from $46,5 \%$ to $72,0 \%$, an extreme average yield of cereals at $60,0 \%$ was observed. At the third trench system, an extreme release of superfine flour at 35,5\% was observed at a general level of $11,9 \%$ to $40,6 \%$ of intermediate products. With an increase in the total product of intermediate milling products on the third trench system from $22,6 \%$ to $47,9 \%$, an extremum of the output of small middlings was observed at $46,4 \%$. Determined extremums are optimal values of intermediate products yield of three break systems.

Conclusions. The given dependences of the output of separate fractions of wheat grain milling products are recommended for calculations of quantitative balances of varietal mills. 


\section{Introduction}

Investigation of wheat grain milling is an actual scientific problem, in connection with the fact that the grain milling function has not been invented due to its complexity $[1-5,8-$ $14,16,19]$.

The function of grain milling is important from the practical point of view, and also necessary for calculating the quantitative balance of the technological process, on the basis of which the calculation of technological equipment of the milling unit and pneumatic transport [6]. Many researchers investigated milling of grain in rollers Campbell G.M., Fistes A., O.Vereschinskii and other researchers. Campbell G.M., Fang C., Muhamad I.I., Webb C., Bunn P.J., Hook S.C.W., Sadhukhan J., Mateos-Salvador F. [1-4, 8, 9, 16, 17] suggested the function of milling wheat grain for the I drowning system, which relates the size of the gap between rollers of the roller machine, the moisture content of the grain and its strength. A. Fistes, G. Tanovic, J. Mastilovic, M. Bardar, A. Takaci, D. Rakic [10-13] proposed a matrix method for calculating granulometric composition of milling products. The function of milling wheat grain on each separate technological system remains unknown [19].

In order to solve the problems of calculating the quantitative balance of grain milling in wheat flour, it is proposed to use dependencies that connect the output of individual fractions of intermediate products from their total product for the first three break systems. These dependencies have a rectilinear form $[15,18]$. In practice, grain milling in rollers is known to reduce the distance between the rollers by shredding large products into small ones.

Linear dependencies between the output of cereals and dunes and the general product of these products are contrary to the phenomenon of grain milling [19].

Taking into account the above, it is relevant to carry out research into the establishment of the output of individual fractions of intermediate products and their total product in the first three break systems.

This will allow the calculation of the output of individual classes of intermediate shredding products when compiling the quantitative balance of milling grain of wheat into varietal flour.

The object of the study is a quantitative assessment of the process of crushing lowviscid grain wheat in the wheat miller.

The output of individual fractions of intermediate shredding products from their general product was investigated.

\section{Materials and methods}

\section{Materials}

During the research wheat grain was processed with the following quality indices: grain weight $-791 \mathrm{~g} / \mathrm{l}$, grain moisture content on the I drought system $-16,2 \%$, vitreousness $-38 \%$, garbage impurity $-0,4 \%$, grain impurity $-2,3 \%$. 


\section{Methods}

Milling of grain. The milling of wheat grain was carried out in the production conditions in rollers, and the technical characteristics of the rollers are given in table. 1 . The technological process was carried out according to the scheme shown in Fig.1

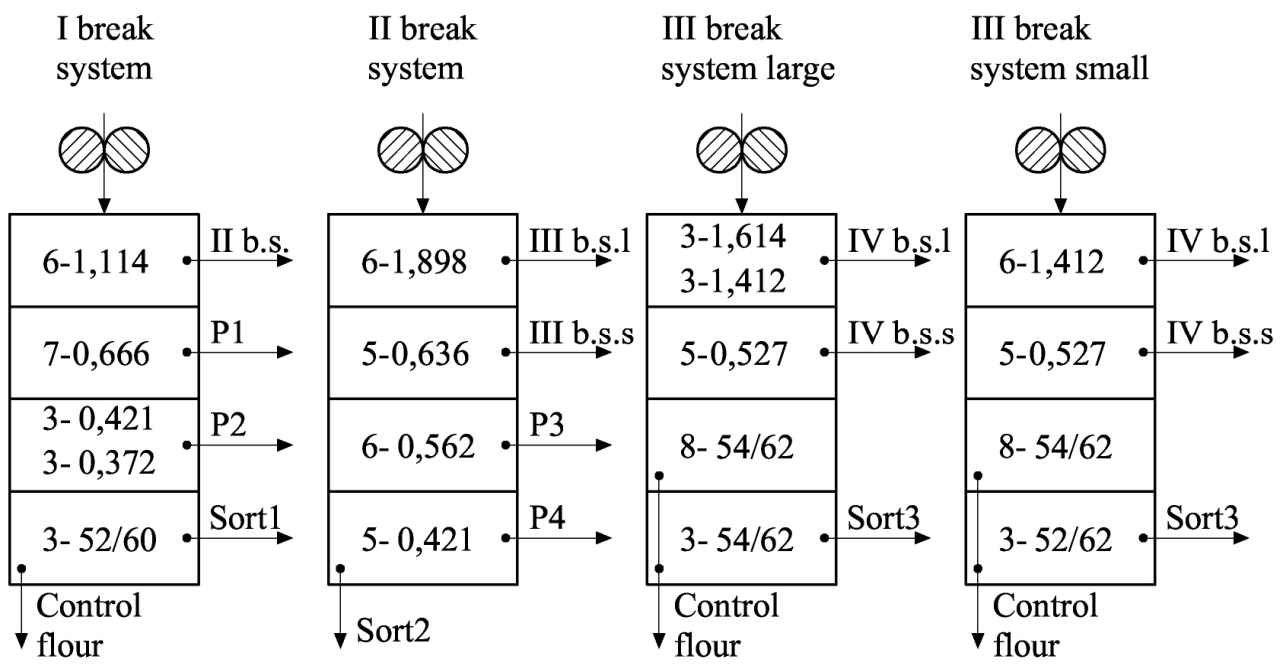

Figure 1. Scheme of tedious process

The formation of intermediate shredding products is carried out according to the following scheme: the technological process of crop formation in wheat mills includes three break systems, and the third break system is divided into large and small ones. The grain is fed to the I system, is crushed and transmitted by pneumo transport to the raiser, which sifted on the sieves.

The first east is sent to the roller mill of the second trample system. The second east in the form of a large gravy goes to the sieve system №1 for enrichment. The third step in the form of an average grits is directed to the sieve system №2 for enrichment. The third approach in the form of a mixture of small grains and dunes is directed to sorting system №1 for division into separate products. The first pass is high quality flour, which is sent to the collecting conveyor.

The crushed product in the roller II of the tidal system is pneumatically transported to the raiser. The first east is fed into a roller mill of the third large system. The second east is fed into a roller mill of the third tiny system of small. The third step in the form of large grains and shells is fed to the sieve system №3 for enrichment. The fourth east as a medium miller is fed to the sieve system №4 for enrichment. A passage in the form of a mixture of small grains, dunes and flour is fed into the raiser of the sorting system number 2 for division into separate products.

The crushed product in the roller II of the tidal system is pneumatically transported to the raiser. The first east is fed into a roller mill of the third large system. The second east is fed into a roller mill of the third tiny system of small. The third step in the form of large grains and shells is fed to the sieve system №3 for enrichment. The fourth east as a medium miller is fed to the sieve system №4 for enrichment. A passage in the form of a mixture of 
small grains, dunes and flour is fed into the raiser of the sorting system number 2 for division into separate products.

The crushed products in the roller system of the third trench of the small system are fed pneumatic transport in the raiser for sorting. The first east is fed into the rolling machine IV of the break system of the large, and the second east is fed into the roller system IV of the break system of fine crushing. The third step in the form of a mixture of medium and small grains, as well as dunts, is fed into the raiser of the sorting system number 3 . The passage gives off flour, which is fed to the control.

The selection of samples of milling products was carried out directly in the technological process as soon as the products passed through the rollers of the rollers.

Kinematical and geometrical parameters of roller work

Table 1

\begin{tabular}{|c|c|c|c|c|c|c|c|}
\hline 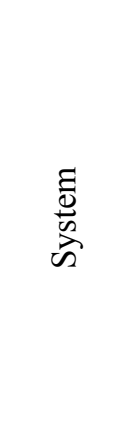 & 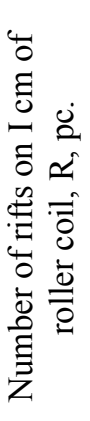 & 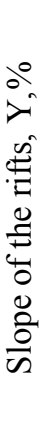 & 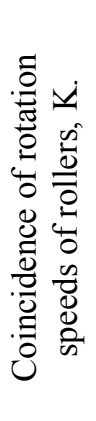 & 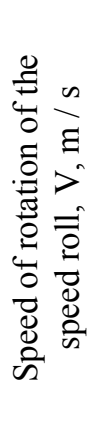 & 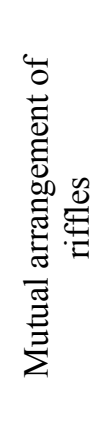 & 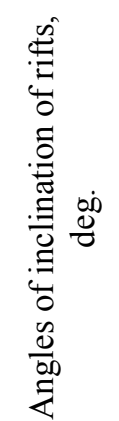 & 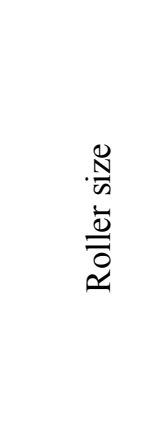 \\
\hline I b.s. & 4 & 6 & 2,5 & 6 & \multirow{4}{*}{$\begin{array}{c}\text { dull to } \\
\text { dull }\end{array}$} & $30^{\circ} / 65^{\circ}$ & $1000 \times 250$ \\
\hline II b.s. & 5,4 & 6 & 2,5 & 6 & & $30^{\circ} / 65^{\circ}$ & $1000 \times 250$ \\
\hline III b.s.l & 6,5 & 6 & 2,5 & 6 & & $30^{\circ} / 65^{\circ}$ & $1000 \times 250$ \\
\hline III b.s.s & 7,5 & 6 & 2,5 & 6 & & $30^{\circ} / 65^{\circ}$ & $1000 \times 250$ \\
\hline
\end{tabular}

Sampling and determination of the general product of shredding products. Changing the milling regimes and sampling were carried out as follows: during the work of the milling unit on each tread system, the entire length of the roller was selected with the help of a tray product in quantities up to $300 \mathrm{~g}$. After the product was selected with a helmet, which is equipped with rollers, they changed the distance between the rollers and repeatedly carried out the selection of the product along the entire length of the roller.

After selecting the shredding products, all selected and weighed samples were sieved on the control sieves to determine the total product of the intermediate products. The selection of milling products was carried out from the third trunks system to the first to avoid the effects of shredding regimes on the next system. For the 1st and 2nd trench systems, sifting of the milling products was carried out on a control metal sheet with the dimensions of the apertures of $1000 \mu \mathrm{m}$, for the third trench of large and small sieving carried out on the control sieve $560 \mu \mathrm{m}$.

The total product of intermediate products of crushing, which characterized the operating mode of the roller was calculated by the formula: 


$$
B_{o}=\left(\frac{m_{n}}{m_{g}}-N\right) \cdot 100
$$

where $B_{o}$ - total product of intermediate shredding products, $\% ; m_{n}$ - is the total mass of the sample after the roller, g; $m_{g}$ - mass of the passage of the control sieve, g; $N-$ shortcomings, $g$.

The shortcomings were determined by taking the chopping products before they arrived on the rolls of the roller. The product after sifting was screened on control sieves, was converted into interest and taken into account when calculating the yield of individual fractions of milling products. In the event that lack of attention was not observed during calculations were not taken into account. The following sieves were used to determine the underdevelopment: for I and II break systems $-1000 \mu \mathrm{m}$, for the third break system -560 $\mu \mathrm{m}$.

Quantitative evaluation and classification of milling products. The passage fraction was weighed after sifting and then scattered on sieves with apertures of $560 \mu \mathrm{m}$, $390 \mu \mathrm{m}, 250 \mu \mathrm{m}, 160 \mu \mathrm{m}$, and $132 \mu \mathrm{m}$. The $132 \mu \mathrm{m}$ sieve was extracted with flour. The yield of individual fractions of crushing products was characterized as the passage and the east of the screen, the dimensions of which holes are shown in table 2

Classification of wheat grain milling products in roller mills

Table 2

\begin{tabular}{|c|c|c|c|}
\hline \multirow{2}{*}{ № } & \multirow{2}{*}{ Product name } & \multicolumn{2}{|c|}{ Particle size range, $\boldsymbol{\mu m}$} \\
\cline { 3 - 4 } & & Pass sieve & East sieve \\
\hline 1 & Large middlings & 1000 & 600 \\
\hline 2 & Medium middlings & 600 & 390 \\
\hline 3 & Small middlings & 390 & 250 \\
\hline 4 & Superfine flour & 250 & 160 \\
\hline 5 & Flour & 132 & - \\
\hline
\end{tabular}

After sifting, each passing fraction of the product was weighed and converted to a percentage by the formula 2 :

$$
B_{i}=\left(\frac{m_{i}}{m_{g}}-N\right) \cdot 100
$$

where, $B_{i}$ - yield of the $\mathrm{i}$-th faction, $\% ; m_{g}-$ is the total mass of the sample after the roller, g; $m_{i}$ - mass of the i-th product obtained after sieving, g; $N$ - shortcomings.

Mathematical and statistical processing. On the basis of the obtained values, the dependencies in coordinates were constructed "the total product of the intermediate shredding products - the yield of the fraction of the product". On the basis of experimental data, using the least squares method, the yield equation of each individual product was 
calculated (large middlings, medium middlings, small middlings, superfine flour, flour) from general products.

Method of determination of quality indices of processed wheat grain. The moisture content of the grain was determined by drying the weight of the crushed product weighing 5 $\mathrm{g}$ for 40 minutes in the drying cabinet of SESH-3M at $130{ }^{\circ} \mathrm{C}[7,20]$.

Humidity of grain $W$ was calculated by the formula:

$$
W=\frac{m_{0}-m_{1}}{m_{0}} \cdot 100
$$

where, $m_{0}$ - weight of weight loss before drying, g.; $m_{1}-$ weight of weight loss after drying, g.

The grain weight was determined on a litter purity by weighing 1 liter of grain.

The total vitreousness was determined by means of a diaphragm, by means of a sightglass eyepiece of a diaphanoscope of 100 grains. To completely glassy grains were counted such that they were completely luminous, and to the mealy - completely not enlightened grains. Grains with partially translucent or partially non-translucent endosperm were attributed to partially glassy grains. General vitality was determined by the formula:

$$
V=C+\frac{P}{2}
$$

where $C$ - number of fully glassy seeds, pcs.; $P$ - the number of partially glassy seeds, pcs.

The common glasswidth was calculated with rounding to an integer.

Smear and grain impurities were determined by sieving weights of $50 \mathrm{~g}$ from the average sample in laboratory sieves of $1,7 \times 20 \mathrm{~mm}$ and 1,0 . After sifting, all ladders were individually dismantled on a collapsible board, separating whole grains, garbage impurities and grain additives separately. Passage was attributed to garbage impurity [19].

\section{Results and discussion}

\section{Yield of the intermediate product and the milling on the 1st drowning system}

Investigations of the outflow of droplets, dunsters and flour on the I-droplet system have shown that with an increase in the total product of intermediate products $(1000 \mu \mathrm{m}$ sieve) from $29,4 \%$ to $56,6 \%$, the yield of these products has a curvilinear polynomial character that is different from those data, which are presented by a number of researchers.

With an increase in total product of intermediate products from $29,4 \%$ to $56,6 \%$, the yield of large grains decreased by $2,6 \%$ from $12,9 \%$ to $10,3 \%$. The research also found that the dependence of the yield of large grains on the total product of intermediate products has an extremum and achieves a maximum value within the limits of $37,0-45,0 \%$, with the yield of large grains respectively ranging from $15,5-15,6 \%$.

The results of the research are shown in Figure 2. The yield of medium, small grains, dunes and flour has a growing nonlinear character with an increase in the total. 
With an increase in the total production of milling products on the I drowning system from $29,4 \%$ to $56,6 \%$, the average grain yield increased by $10,1 \%$ from $7,9 \%$ to $18,0 \%$; the yield of small grains increased by $3,8 \%$ from $2,4 \%$ to $6,2 \%$; the yield of dunstids increased by $8,7 \%$ from $3,9 \%$ to $12,6 \%$, and yield of flour increased by $7,3 \%$ from $2,2 \%$ to $9,5 \%$.

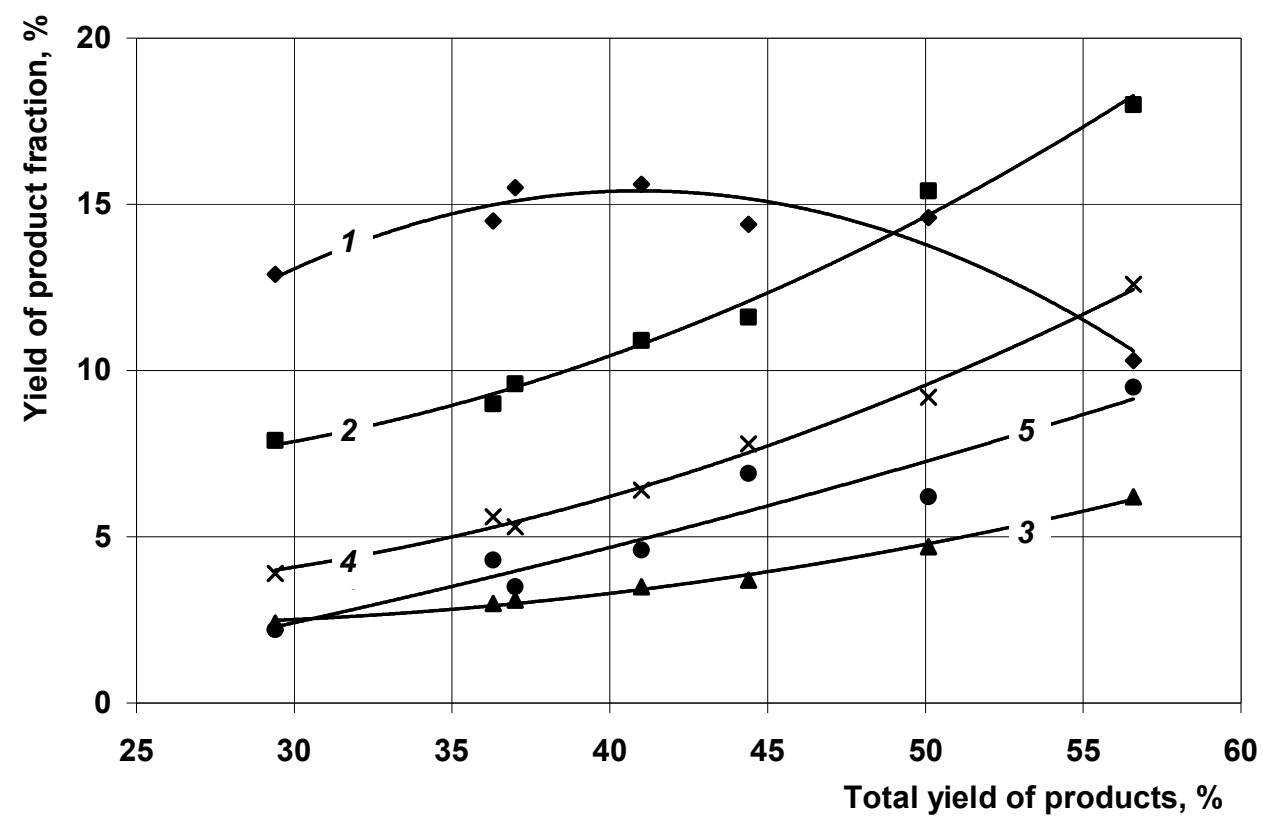

Figure 2. Yield middlings, superfine flour and flour depending on the mode of operation of the first break system:

1 - large middlings; 2 - medium middlings; 3 - small middlings; 4 - superfine flour; 5 -flour.

The presence of the extremum of the curve of the exit of the large middlings has the following explanation: an increase in the yield of the medium middlings, small middlings, superfine flour and flour is due to the redistribution of large grains into smaller milling products with an increase in the total product of the products on the 1st droplet system, as evidenced by the declining nature of the curve of the exit of the large grains with the general product of milling products more than $40 \%$ and the growing nature of the curves, which describe the yield of all other grain milling products of wheat on the I-ration system.

The mathematical processing of the experimental data obtained from the yield of intermediate shredding products on the I-droplet system made it possible to establish equations that describe the yield of individual product fractions from their total product:

Yield of large middlings:

$$
B_{l m}=-0,0196 B^{2}+1,605 B-17,45
$$

Yield medium middlings:

$$
B_{m m}=0,00805 B^{2}-0,305 B+9,79
$$


Yield of small middlings:

$$
B_{s m}=0,0034 B^{2}-0,163 B+4,3
$$

Yield of superfine flour:

$$
B_{s f}=0,0061 B^{2}-0,215 B+5,05
$$

Yield of flour:

$$
B_{f}=0,0016 B^{2}+0,113 B-2,44
$$

where, $B_{l m}$ - yield of large middlings, $\% ; B_{m m}$ - yield of the medium middlings, $\% ; B_{s m}$ yield of small middlings, $\% ; B_{s f}$ - yield of the superfine flour, $\% ; B_{f}$ - yield of flour, $\% ; B$ - is the total product on the system (1000 $\mu \mathrm{m}$ sieve passage), $\%$.

The same kind of dependence was obtained by Vereshchinsky O.P. [19] when milling wheat grain in a laboratory roller mill with a roller diameter of $185 \mathrm{~mm}$ (Figure 3), which confirms the objectivity of the research.

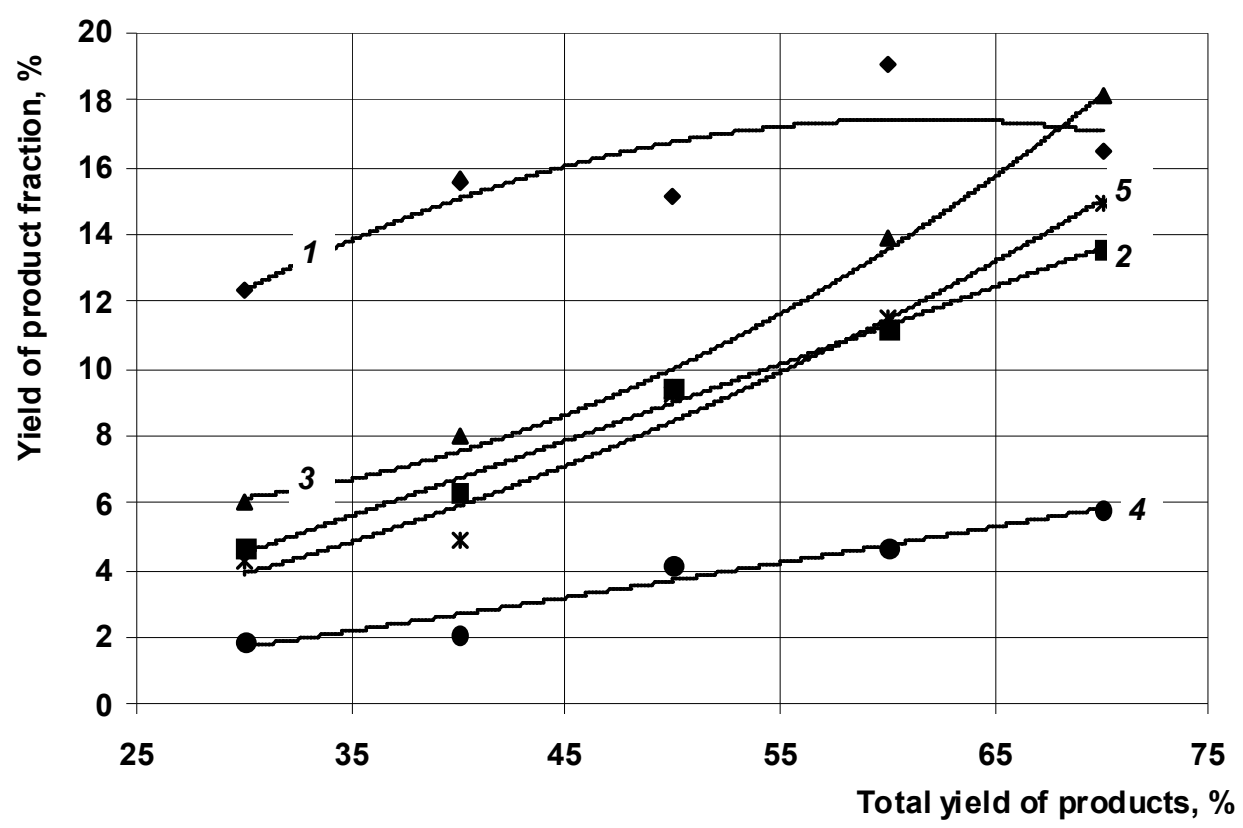

Figure 3. Yield of intermediate shredding products in a laboratory roller mill [19]:

1 - large middlings; 2 - medium middlings; 3 - small middlings; 4 - superfine flour; 5 - flour.

Figure 2 and 3 indicate the nonlinear nature of the dependence of the yield of individual intermediate shredding products on their overall product when chopping in a roller machine on the 1 st droplet system. 


\section{Yield of intermediate products and milling on the second tread system}

Investigations of the operating regime of the II tram system have established that with an increase in the total product of intermediate products from $46,5 \%$ to $72,0 \%$, the yield of large, medium grains and flour is nonlinearly increasing, while the yield of small middlings and superfine flour is increased with increasing production of total products on II from $46,5 \%$ to $72,0 \%$. The results of the research are shown in Figure 4.

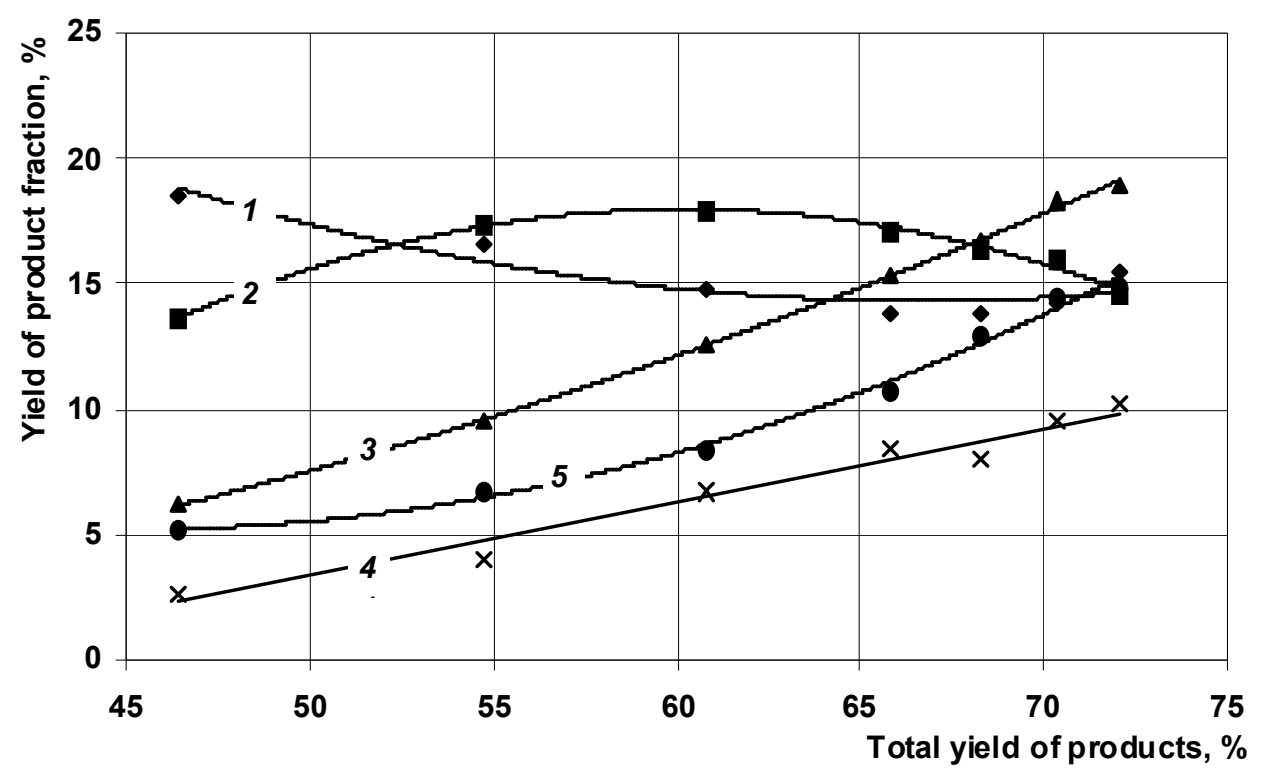

Figure 4. Yield middlings, superfine flour and flour depending on the milling mode of the second break system:

1 - large middlings; 2 - medium middlings; 3 -small middlings; 4 -superfine flour; 5 -flour.

With the increase in the total product of shredding products from $46,5 \%$ to $72,0 \%$ (1000 $\mu \mathrm{m}$ sieve) on the second trench system, the yield of large middlings decreased by $3,0 \%$, from $18,5 \%$ to $15,5 \%$. From the given Figure 4, it can be seen that with the increase in the total product of milling products on the second trench system, the yield of large grains decreases steadily, and the yield of small middlings, superfine flour and flour is constantly increasing.

The yield of the medium middlings with the increase in the total product of intermediate milling products in the specified limits varied from $13,6 \%$ to $14,6 \%$, while the dependence of the yield of the average middlings has an extremum, which allows to determine the milling mode, which achieves the largest yield of the average middlings. The presence of an extremum within the overall value of $60 \%$ suggests that the increase in the total product of milling products above $60 \%$ leads to the redistribution of not only large grains into smaller products (small middlings, superfine middlings and flour) but also the medium middlings. 
The yield of small middlings with an increase in the total product of intermediate shredding products from $46,5 \%$ to $72,0 \%$ increased by $12,8 \%$ from $6,2 \%$ to $18,9 \%$, and the yield of superfine flour increased by $7,5 \%$, namely from $2,7 \%$ to $10,2 \%$.

With an increase in the total product of intermediate shredding products from $46,5 \%$ to $72,0 \%$, the flour yield increased by $9,7 \%$ from $5,2 \%$ to $14,9 \%$.

In studies of the mode of operation of the II system, the operating mode of the I system was $37,9 \%$ on average.

On the basis of experimental data, dependences were obtained that describe the yield of individual fractions of the milling products of the II tram system from their total product:

Yield of large middlings:

$$
B_{l m}=0,0111 B^{2}-1,48 B+63,66
$$

Yield medium middlings:

$$
B_{m m}=-0,0228 B^{2}+2,7501 B-64,91
$$

Yield of small middlings:

$$
B_{s m}=0,508 B-17,9
$$

Yield of superfine flour:

$$
B_{s f}=0,298 B-11,6
$$

Yield of flour:

$$
B_{f}=0,014 B^{2}-1,27 B+34,09
$$

where $B_{l m}$ - yield of large middlings, $\% ; B_{m m}$ - yield of the medium middlings, $\% ; B_{s m}-$ yield of small middlings, $\% ; B_{s f}$ - yield of the superfine flour, $\% ; B_{f}$ - yield of flour, $\% ; B$ - is the total product on the system (1000 $\mu \mathrm{m}$ sieve passage), $\%$.

\section{Yield of intermediate products and milling on the third trench system}

On the third break system, the first east of the second trench system, which contains a significant amount of endosperm, is crushed. The crushed product consists of medium middlings, small middlings, superfine flour and flour. Large middlings were not found in shredding products. The research has established that with an increase in total product (560 $\mu \mathrm{m}$ sieve passage) from $11,9 \%$ to $40,6 \%$, the yield of the product, which was classified as an average grains, increased by an average of $15,5 \%$ from $1,9 \%$ to $17,4 \%$, the yield of small middlings decreased by $3,1 \%$ from $2,6 \%$ to $5,7 \%$, the yield of superfine flour increased by an average of $2,4 \%$ from $1,7 \%$ to $4,1 \%$, and the yield of flour increased by an average of $7,4 \%$ from $5,4 \%$ to $12,8 \%$.

From the given Figure 5 it can be seen that the yield of the medium middlings, superfine flour and flour, depending on the general product of the milling products on the third break system, is of a nonlinear nature, and the yield of small middlings is linear. From 
the given Figure 5 it can be seen that with an increase in total production from $11,9 \%$ to $40,6 \%$, the yield of superfine flour has an extremum with a total product of crushing products $35,5 \%$.

During investigations of the operating modes of the III tidal system of large and small, the average product of intermediate products of crushing II tram system was $64,3 \%$.

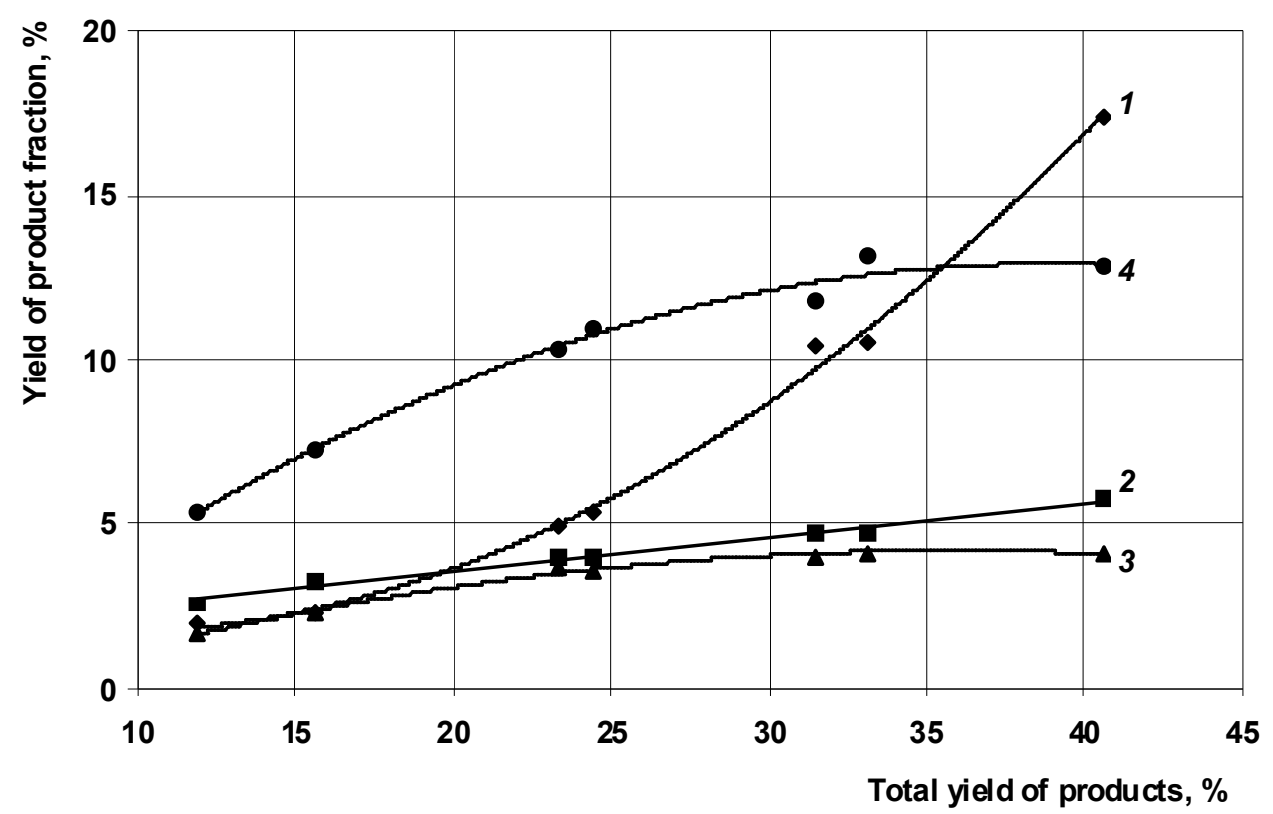

Figure 5. Yield middlings, superfine flour and flour depending on the milling mode of the third break system of large:

1 - medium middlings; 2 - small middlings; 3 -superfine flour; 4 -flour

On the basis of experimental data the dependences of yield of separate fractions of milling products of the third break system of large of their general product were obtained:

Yield medium middlings:

$$
B_{m m}=0,0154 B^{2}-0,26 B+2,74
$$

Yield of small middlings:

$$
B_{s m}=0,101 B+1,51
$$

Yield of superfine flour:

Yield of flour:

$$
B_{s f}=-0,0045 B^{2}+0,32 B-1,54
$$




$$
B_{f}=-0,0104 B^{2}+0,81 B-2,77
$$

where $B_{m m}$ - yield of the medium middlings, $\% ; B_{s m}$ - yield of small middlings, $\% ; B_{s f}$ yield of the superfine flour, $\% ; B_{f}$ - yield of flour, $\% ; B$ - is the total product on the system (560 $\mu \mathrm{m}$ sieve passage), $\%$.

Investigations of the milling regimes of products on the third trench system of small ones showed that with an increase in the total product of intermediate shredding products from $22,6 \%$ to $47,9 \%$, the yield of the average grits increased by $2,3 \%$ from $14,0 \%$ to $16,3 \%$, and in the general product of $33,1 \%$ there is an extremum of the function with a minimum value at which the yield of the average cream was $13,6 \%$. The yield of small middlings increased by $8,0 \%$ from $3,3 \%$ to $11,3 \%$, the yield of superfine flour increased by $7,2 \%$ from $1,4 \%$ to $8,6 \%$, while the yield of flour increased by $8,2 \%$ from $3,8 \%$ to $12,0 \%$. The results of the study of the yield of circular dendrobates and flour during the milling of stair products on the 3rd drowning system are given in Figure 6.

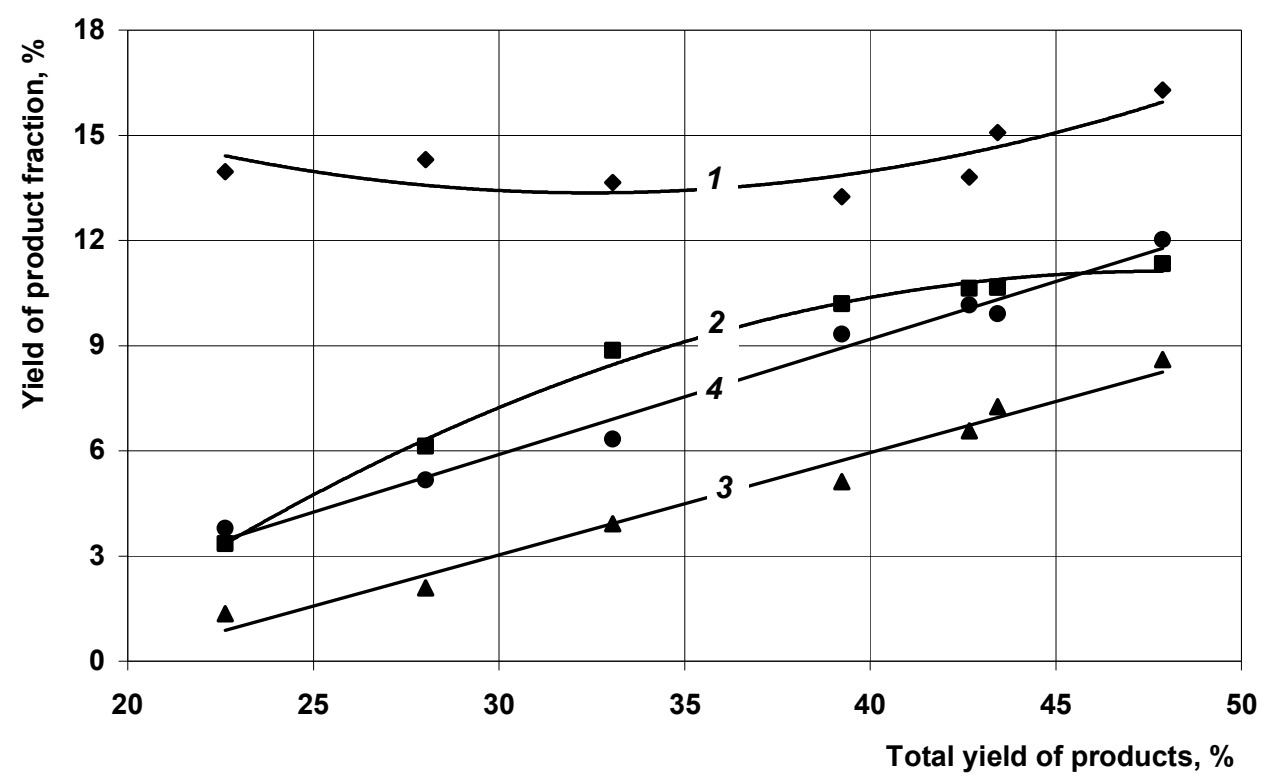

Figure 6. Yield middlings, superfine flour and flour depending on the milling mode of the third break system of small:

1 - medium middlings; 2 -small middlings; 3 - superfine flour; 4 - flour.

With an increase in the total product of intermediate products and milling from $22,6 \%$ to $47,9 \%$, the yield of small middlings had an extreme of $46,4 \%$.

From the given Figure 6 it can be seen that the dependence of the yield of medium and small middlings, depending on the general product of the products on the third trench system, is of a nonlinear nature, and the dependence of the yield of superfine flour and flour is linear in nature. 
The processing of experimental data allowed us to obtain equations that describe the yield of individual fractions of milling products on the third trench system of small ones from their total product:

Yield medium middlings:

$$
B_{m m}=0,0112 B^{2}-0,73 B+25,31
$$

Yield of small middlings:

$$
B_{s m}=-0,0128 B^{2}+1,19 B-17,25
$$

Yield of superfine flour:

$$
B_{s f}=0,29 B-5,68
$$

Yield of flour:

$$
B_{f}=0,32 B-3,91
$$

where, $B_{m m}$ - yield of the medium middlings, $\% ; B_{s m}$ - yield of small middlings, $\% ; B_{s f}-$ yield of the superfine flour, $\% ; B_{f}$ - yield of flour, $\% ; B$ - is the total product on the system (560 $\mu \mathrm{m}$ sieve passage), $\%$.

Comparing the results of studies with similar data from other researchers $[15,18]$, it can be seen that the yield of intermediate shredding products of the first three break systems has a nonlinear character for most middlings products, which confirms the crushing of large particles in the finer ones in the process of milling grain in flour.

\section{Conclusions}

The yield of intermediate milling products from their total product for many products of the first three break systems has a nonlinear character. The mathematical dependences of the yield of individual fractions of the milling products of the first three break systems from their total product are obtained. It is recommended to use them for the development of quantitative balances of milling of low-viscid grain wheat.

The scientific novelty consists in deepening the theory of milling of low-viscid grain of wheat in the case of varieties of wheat mills, as well as substantiation of the change of the yield of separate fractions of intermediate products of milling from the general product of these products on the first three break systems according to the curvilinear law. 


\section{References}

1. Campbell G.M., Sharp C., Wall K., Mateos-Salvador F., Gubatz S., Hutty A., Shewry P. (2012), Modelling wheat breakage during roller milling using the double normalised Kumaraswamy breakage function: Effects of kernel shape and hardness, Journal of Cereal Science, 55, pp. 415-425.

2. Campbell G.M., Webb C. (2001), On predicting roller milling performance. Part I: the breakage equation, Powder Technology, 115, pp. 234-242.

3. Campbell G.M., Bunn P.J., Webb C., Hook S.C.W. (2001), On predicting roller milling performance. Part II: the breakage function, Powder Technology, 115, pp. 243-255.

4. Campbell G.M., Fang C., Muhamad I.I. (2001), On predicting roller milling performance VI: Effect of kernel hardness and shape on the particle size distribution from first break milling of wheat, Trans IChemE, 79(C), pp. 211-218.

5. Dmitruk Y., Vereschinskii O., Kharchenko Y. (2013), Efficiency increase the milling systems in the bread baking wheat millings, Ukrainian Food Journal, 2(2), pp. 163-168.

6. Dmitruk Y., Ilchuk V., Kharchenko Y., Boretz A. (2009), Add the optimal quantitative balance of milling grain - mean to orient on the effective work of the milling department, Grain and bread, 1, pp. 26-27.

7. Dowell F.E. (2000), Differentiating vitreous and non-vitreous durum wheat kernels by using near-infrared spectroscopy, Cereal Chem, 77(2), pp. 155-158.

8. Fang C., Campbell G.M. (2002), Effect of roll fluting disposition and roll gap on breakage of wheat kernels during first-break roller milling, Cereal Chem, 79(4), pp. 518-522.

9. Fang C., Campbell G.M. (2003), On predicting roller milling performance IV: Effect of roll disposition on the particle size distribution from first break milling of wheat, Journal of Cereal Science, 37, pp. 21-29.

10. Fistes A., Tanovic G. (2006), Predicting the size and compositional distributions of mheat flour stocks following first break roller milling using the breakage matrix approach, Journal of Food Engineering, 75, pp. 527-534.

11. Fistes A., Tanovic G., Mastilovic J. (2008), Using the eight-roller mill on the front passages of the reduction system, Journal of Food Engineering, 85, pp. 296-302.

12. Fistes A., Rakic D., Takaci A., Brdar M. (2013), Using the breakage matrix approach to define the optimal particle size distribution of the input material in a milling operation, Chemical Engineering Science, 102, pp. 346-353.

13. Fistes A., Rakic D., Takaci A., Brdar M. (2014), Solution of the breakage matrix reverse problem, Powder Technology, 268, pp. 412-419.

14. Kharchenko Y., Perehuda N., Shnipko I. (2015), Granulometric composition of intermediate products of milling with two-stage milling of wheat grain in laboratory conditions, Grain storage and processing, 6-7, pp. 62-64.

15. Martinenko Y.F., Chebotaryov O.N. (1992), Designing of flour-milling and cereal plants with the basics of CAD, Agropromizdat, Moscow.

16. Mateos-Salvador F., Sadhukhan J., Campbell G.M. (2013), Extending the normalised Kumaraswamy breakage function for roller milling of wheat flour stock to second break, Powder Technology, 237, pp. 107-116.

17. Mateos-Salvador F., Sadhukhan J., Campbell G.M. (2011), The normalised Kumaraswamy breakage function: A simple model for wheat roller milling, Powder Technology, 208, pp. 144-157. 
18. Ptuskin A.T., Novitskiy O.A. (1979), Automation of production processes in the grain storage and processing industry, Kolos, Moscow.

19. Vereshchynsky A.P. (2013), Scientific basis and practice of increasing efficiency of varietal bread wheat milling, NUFT, Kyiv.

20. Samson M.F., Mabille F., Chéret R., Abécassis J., More M.H. (2005), Mechanical and physicochemical characterization of vitreous and mealy durum wheat endosperm, Cereal Chem, 82(1), pp. 81-87. 